\title{
Evolución de la participación de los escolares en las finales de cross regional durante tres temporadas
}

\author{
Participation trends of children in regional \\ cross-country final during three seasons
}

\author{
Vílchez, M.P. y Molina, J.M. ${ }^{2}$ \\ 1 Universidad Católica San Antonio de Murcia (UCAM) y Directora Técnica de la federación de triatlón de la Región de Murcia (TRIMURCIA). \\ 2 Universidad Católica San Antonio de Murcia (UCAM) y Presidente de la federación de atletismo de la Región de Murcia (FAMU). \\ Recibido: 03/03/2014; Aceptado: 28/11/2014.
}

\begin{abstract}
Resumen: El deporte escolar es una buena estrategia de promoción deportiva, que gracias a los programas de promoción de actividad física en edad escolar, está aumentando en los últimos ańos (CSD, 2009; Valovich et al., 2011) y, concretamente, el cross escolar de atletismo es una modalidad que por sus características puede aumentar esta participación. Para tal fin, en el presente trabajo se analiza la participación de los escolares en las finales de triatlón escolar de las temporadas 2011, 2012 y 2013 para conocer las características de esta participación escolar y poder orientar las tareas de promoción deportiva por parte de las entidades correspondientes. La muestra se compuso de 4205 participaciones del cross escolar durante las temporadas 2010-11, 2011-12 y 2012-13. Del total de la muestra, 51.4\% fueron chicos y el $48.6 \%$ chicas. Para el análisis de los datos se utilizó el SPSS v.21. Según los resultados, se está realizando una gran labor de participación en las chicas, teniendo que hacer más hincapié en las categorías superiores y en los centros concertados y privados de Murcia. Además, se necesitan más estudios que analicen esta participación para seguir orientando los programas de promoción deportiva.

Palabras clave: Atletismo, escolar, participación, promoción deportiva.
\end{abstract}

Abstract: Sports at school age are a useful tool of sports promotion, which its participation is growing (CSD, 2009; Valovich et al., 2011). Concretely, cross-country running is a sport that has simple characteristics to be able to improve that promotion. For this reason, in the following study, the participation of scholars on cross-country events from seasons 2010-11, 2011-12 and 2012-13 is analyzed to know the characteristics of scholar participation and to be able to guide promotion sports tasks of corresponding entities. A sample of 4205 participations of scholars on cross-country events was used. From the total sample, $51.4 \%$ were boys and $48-6 \%$ were girls. Data analysis was made using statistics programme SPSS, version 21. It was concluded that it has been developed a great promotion tasks between girls, however, there exists a need to improve sports promotion programmes in older scholars and also among private schools.

Key words: Triathlon, scholars, participation, sport promotion.

\section{Introducción}

En el artículo 10 de la Ley (Comunidad Autónoma de Murcia) 2/2000, de 12 de julio, del Deporte de la Región de Murcia, se define el deporte escolar como "actividad deportiva organizada, practicada por los escolares de nuestra Región en horario no lectivo" (p. 6). El atletismo, concretamente el cross escolar, cumple con uno de los principios importantes como deporte para la promoción del deporte escolar, indicados en la vigente Ley, garantizando que todos los escolares puedan conocer bien la práctica de esta modalidad deportiva (dada la sencillez de "salir a correr"), de acuerdo con su aptitud física y edad (pues en el cross escolar cada alumno va a su ritmo y compite contra él mismo, no como en un deporte de equipo que sus compañeros dependan de su habilidad o su condición física para seguir el juego, por otra parte, tampoco necesita a más compañeros para poder participar en el cross). Dada

Dirección para correspondencia [Correspodence address]: M. P. Vílchez. Departamento de Educación, Universidad Católica San Antonio de Murcia (UCAM). Campus de Los Jerónimos, s/n. 30107, Guadalupe, Murcia (España).E-mail: pvilchez@ucam.edu que la finalidad del deporte escolar es completar el desarrollo educativo de cada deportista, a la vez que satisfacer sus necesidades individuales, se considera que el cross escolar está en una posición privilegiada para promocionar la participación deportiva en edad escolar.

Según el Consejo Superior de Deportes (CSD, 2009) en el Plan Integral para la Actividad Física y el Deporte, la participación deportiva en edad escolar está aumentando en los últimos años en España y según Valovich et al. (2011) también en el ámbito internacional. Sin embargo, aunque hay muchos programas de promoción deportiva y un fácil sistema de inscripción escolar, es necesario analizar esta participación deportiva, en términos reales dentro de cada modalidad deportiva, para conocer las características, la evolución y proponer estrategias de promoción y mejora de estos programas, adaptados a la realidad de la participación escolar.

En el caso del atletismo, según un estudio de los hábitos deportivos de la población escolar en España del Consejo Superior de Deportes (CSD, 2011), el salir a correr es la 
tercera actividad no organizada más practicada por los escolares, y en cambio, el atletismo como actividad organizada solo lo practican un 3\% de escolares. Estos datos coinciden con otros estudios previos, por ejemplo, Chillón, Tercedor, Delgado y González-Gross (2002) encontraron que practicaban atletismo un 3,4\% de los escolares. No obstante, el cross escolar es una modalidad dentro del atletismo que permite ser entrenado fuera de la pista sin requerir más material que, simplemente, salir a correr, de ahí la el interés de conocer las condiciones y evolución de la participación de esta prueba escolar en las últimas temporadas en la Región de Murcia para tomar medidas en cuanto a su promoción deportiva.

En cuanto al género, según un estudio de Luengo (2007) en la Comunidad de Madrid, la participación de las chicas en actividades extra-escolares a esta edad fue sustancialmente menor que la de los chicos, incluso con diferencias más acusadas en otros estudios (Chillón, Tercedor, Delgado y González-Gross, 2002), por lo que se tendrá en cuenta el género a la hora de analizar la participación en el cross escolar de la Región de Murcia.

También es importante tener en cuenta que todavía en edad escolar pero en las categorías superiores suele haber un abandono de la práctica deportiva, como indica Mollá (2007). Por eso, se considera importante analizar la evolución de la participación a lo largo de las temporadas para conocer si los escolares se mantienen en esta práctica deportiva una vez captados.

Es de tanto interés esta promoción, con el objetivo aumentar la participación deportiva en edad escolar, que numerosos autores han estudiado las diferentes motivaciones de los niños hacia la práctica deportiva (Bailey, Cope y Pearce, 2013). Si se tiene en cuenta las motivaciones de los jóvenes (las que tendrán los escolares en un futuro), el atletismo o cross cumpliría con características de estas motivaciones señaladas por Moreno, Martínez y Alonso (2006) tanto en las chicas como actividad de tipo individual y en los chicos como actividad de carácter competitivo.

También suele existir la polémica sobre a qué edad se produce la especialización deportiva y/o edad a la que los niños pueden competir, por eso, la citada Ley del Deporte de la Región de Murcia, explicita que debe tener un carácter polideportivo y no exclusivamente competitivo. En relación a esto, autores como Norris (2011) recomiendan que los niños de 8-9 años de edad ya deben aprender a entrenar, pasando a un entrenamiento dirigido más a la competición a partir de los 15-16 años. Esta recomendación coincide con estudios internacionales sobre la carrera de fondo y medio fondo cuando Gorgan y Rata (21013) afirman que a los 7-8 ya la categoría puede ser competitiva, siendo a partir de la categoría de 12-13 ańos cuando se busca rendimiento deportivo y la intensidad puede ir aumentando considerablemente.

Al tratarse de atletismo y la modalidad de cross, que supo- ne "solo correr" se tendrá en cuenta desde la categoría benjamín, dado que es una habilidad motriz básica y coordinativa que está recomendada desde los seis años (Romero, 2001, citado en González, García-López, Contreras y Sánchez-Mora, 2009).

En el caso de la Región de Murcia, de la promoción deportiva del cross escolar se encarga la Federación de Atletismo de la Región de Murcia (FAMU). Esta federación ha desarrollado diferentes programas en los últimos años de promoción, como el programa PROADES (del CSD) o "jugando al atletismo". Hasta 2012, no estaba permitido que los escolares federados participaran en el cross escolar, normativa que se ha cambiado incrementando la participación en el cross en los últimos años.

Por todo ello, en el presente trabajo se analiza la participación de los escolares en las finales del cross escolar de las temporadas 2010-2011, 2011-12 y 2012-13, en función de la categoría, el género y el tipo de centro, realizando un análisis de la evolución de esta participación y las posibles repercusiones que esta nueva normativa tiene a nivel de deporte escolar.

\section{Método}

\section{Muestra}

Se registraron 4205 participaciones del cross escolar durante las temporadas 2010-11, 2011-12 y 2012-13, donde 1354 participaciones (32.2\%) fueron de 2011, 1429 participaciones (34.0\%) de 2012 y 1422 participaciones (33.8\%) de 2013. Del total de la muestra, $51.4 \%$ fueron chicos y el $48.6 \%$ chicas.

Los escolares fueron clasificados en la categoría benjamín (23.8\%), alevín (24.6\%), infantil (22.1\%), cadete (16.3\%) y juvenil (13.2\%). Se registraron las participaciones en las temporadas 2011 y 2012 en función del centro educativo, siendo de centros públicos $(73.4 \%)$, concertados $(24.7 \%)$ y privados (2\%). En la última temporada, se tuvo en cuenta las diferentes jornadas de cross en edad escolar ya que cambió el formato de la competición; de esta manera, se obtuvo el total de la muestra de 2013 , compitiendo en la primera un $5.8 \%$, en la segunda un $5.1 \%$, en la tercera $5.3 \%$, en la cuarta $6.2 \%$, en la quinta un $5.1 \%$ y en la sexta un 6.3\%. Para el análisis de la participación de los escolares, se obvia que este nuevo formato de competición en la última temporada 2013 pueda influir en la participación de los escolares del cross regional, siendo este cambio de la propia competición, una posible limitación del presente estudio, pero se asume que no hay otra forma de medir la participación en 2013.

\section{Procedimiento}

Se pidió a la secretaría técnica de la Federación de Atletismo de la Región de Murcia (FAMU) la relación de inscripciones 
y clasificaciones de las tres últimas temporadas del cross escolar. Los datos fueron codificados en una base de datos para su posterior análisis.

\section{Análisis de datos}

Se realizaron análisis descriptivos estadísticos a partir de las clasificaciones facilitadas por la FAMU, a través de tablas de contingencia utilizando chi cuadrado, con el paquete estadístico SPSS v. 21. Se tuvo en cuenta una significancia cuando p era $\leq .05$.

\section{Resultados}

La evolución de la participación de los escolares a lo largo de las tres temporadas es bastante equitativa. Aumentó de 2011 a 2012 en un $1.8 \%$ y disminuyó en 2013 con respecto a 2012 un $0.20 \%$. Sin embargo, como se aprecia en la figura 1 , sí se observa una involución de la participación en el cross escolar con la edad en todas las temporadas $\left(\chi^{2}=165.01 ; \mathrm{p}<.001\right)$.

Según la categoría, en la temporada 2011, la mayor participación se produce en la categoría alevín, con un 9.5\%, siendo algo inferior en la categoría benjamín con un 7.6\% y disminuyendo progresivamente la participación conforme avanzan en edad, con un $6.6 \%$ de infantiles, un $5.3 \%$ de cadetes y un 3.3\% de juveniles. En la temporada 2012 la mayor participación se produce en la categoría benjamín con un $10.9 \%$, seguido por orden el resto de categorías, la alevín con un $7.7 \%$, la infantil con un $6.4 \%$, la cadete con un $5.3 \%$ y la juvenil con un $3.8 \%$. En la temporada 2013, se equipara un poco la participación por categorías encontrando diferencias muy significativas ( $\chi^{2}=165.01 ; \mathrm{p}<.001$; figura 1); la más numerosa es la infantil con $9.2 \%$, seguida de la alevín con un $7.5 \%$, de la juvenil con un $6.1 \%$ y, por último, de la cadete y benjamín, con un $5.7 \%$ y $5.3 \%$, respectivamente.

Figura 1. Evolución de la participación en función de las categorías en las finales de cross escolar a lo largo de las temporadas 2010-11, 2011-12 y 2012-13.

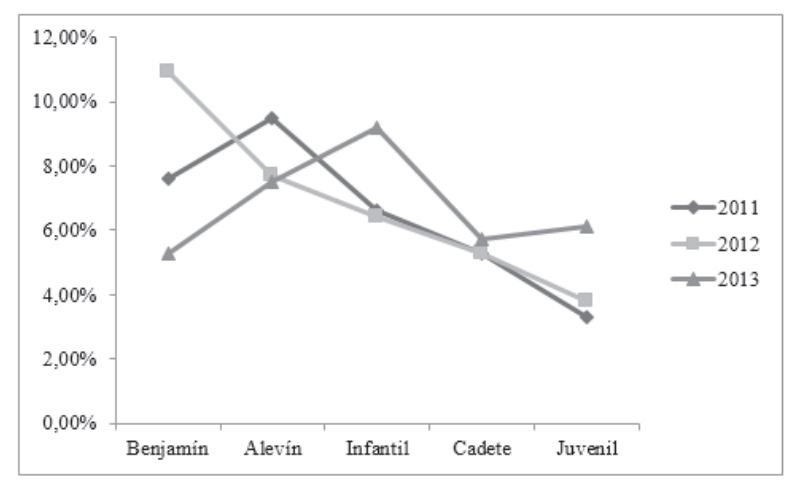

Si se observa la participación solo en la temporada 2011, se observa una tendencia en ambos géneros disminuyen la participación conforme avanzan en edad, siendo mayor la participación en los chicos en categorías superiores de la edad escolar y más equitativa en edades inferiores, incluso un poco mayor en chicas en la categoría alevín $\left(\chi^{2}=8.61 ; \mathrm{p}=.072\right)$. Pero ya en la temporada 2012 se mantiene la misma tendencia en mayores proporciones. En las categorías inferiores es mayor la participación de las chicas, incluso doblando la participación de los chicos en la categoría benjamín (21.3\% de chicas frente al $10.7 \%$ de chicos), y a partir de la categoría infantil la participación de los chicos es superior a la de las chicas, con diferencias muy altamente significativas $\left(\chi^{2}=65.84 ; \mathrm{p}<.001\right)$. En la temporada 2013 solo la categoría infantil tiene más participación de chicas (15\%) que chicos (12\%), mientras que el resto de categorías predomina la participación de los chicos por encima de las chicas $\left(\chi^{2}=29.13 ; \mathrm{p}<.001\right)$. La evolución por género y categoría en cada una de las temporadas se puede observar en la figura 2.

Figura 2. Participación por género a lo largo de las categorías en cada una de las temporadas.

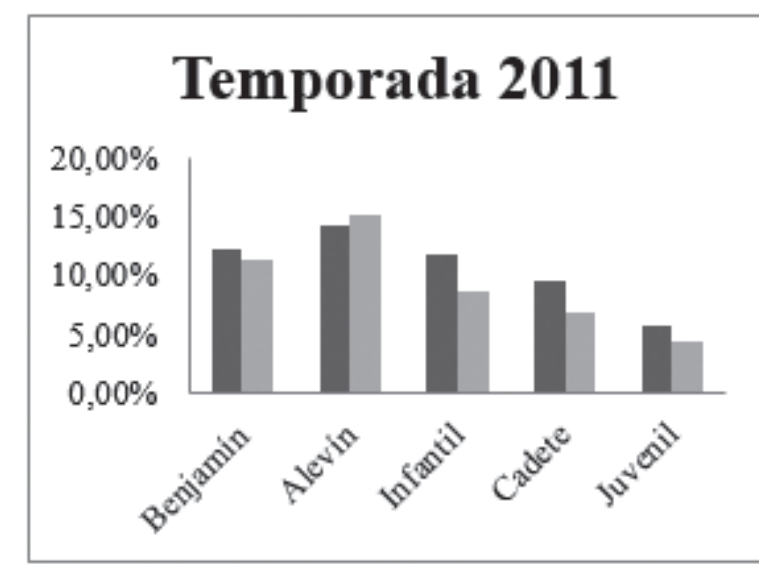

\section{Temporada 2012}

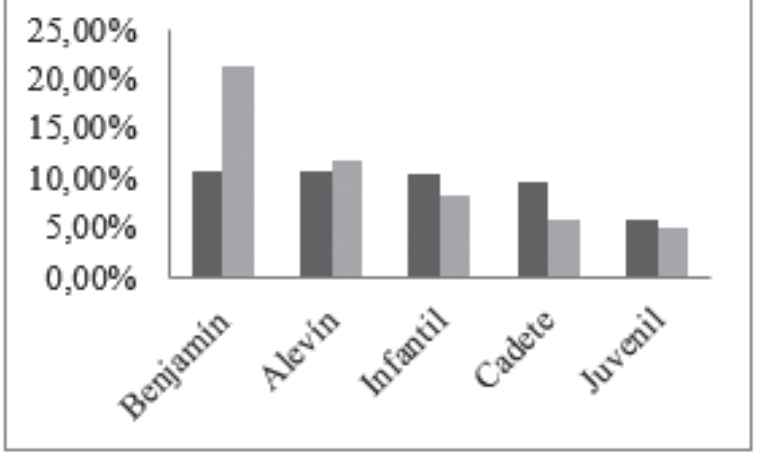




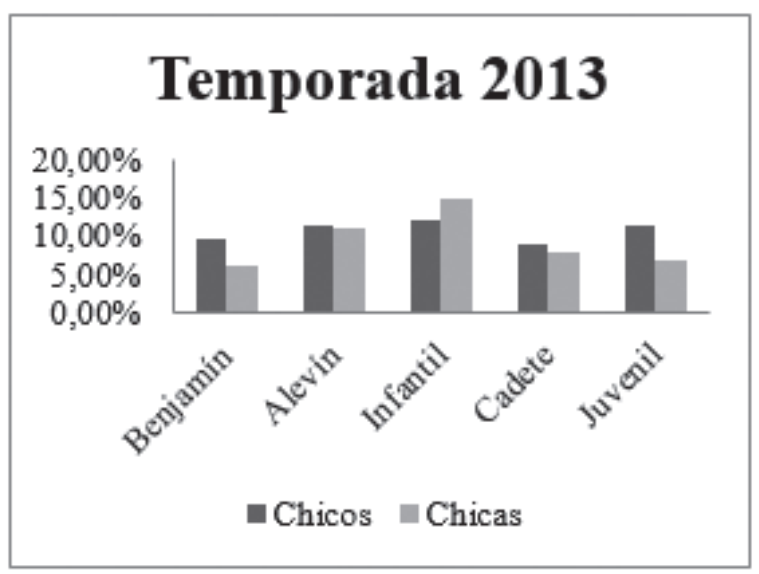

Según el género, la participación es equilibrada a lo largo de las temporadas. En 2011, participaron más chicos que chicas (17.1\% frente al 15.1\%), en 2012 más chicas que chicos en menor proporción (17.8\% frente al $16.2 \%)$ y en 2013 volvieron a participar más chicos que chicas (18\% frente al 15.8\%) ( $\chi^{2}$ $=12.39 ; \mathrm{p}=.002$; figura 3).

Figura 3. Participación en la final de cross escolar en función del género a lo largo de las tres últimas temporadas.

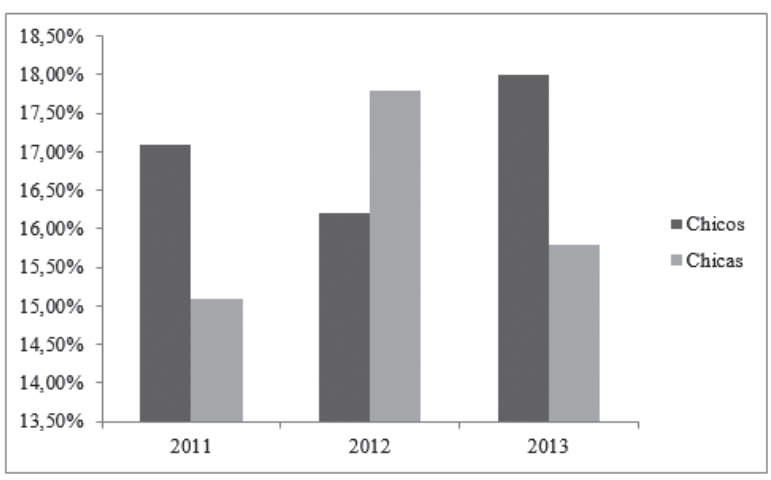

Aunque la distribución de chicos y chicas de la muestra sea equitativa, las categorías superiores tienen mayor participación de los chicos mientras las categorías inferiores tienen mayor participación de las chicas. Concretamente, en la categoría benjamín, las chicas son un 13\% frente al 10.8\% de chicos, siguen siendo más chicas en alevín con $12.6 \%$ frente al $12.1 \%$ de chicos, ya en la categoría infantil se revierte esta tendencia con más participación de chicos (11.4\%) que chicas (10.7\%). En la categoría cadete más mayor participación de chicos que chicas con un $9.4 \%$ frente al $6.9 \%$, al igual que pasa en la categoría juvenil con $7.7 \%$ de chicos y $5.5 \%$ de chicas. Esta tendencia en función de la categoría es altamente significativa $\left(\chi^{2}=37.99 ; \mathrm{p}<.001\right.$; figura 4$)$.
Figura 4. Comportamiento de participación en función del género a lo largo de las categorías en las finales de cross escolar en todas las temporadas.

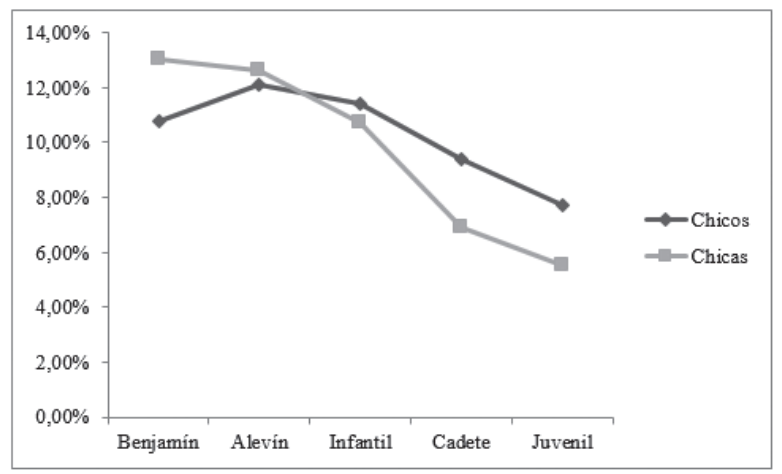

Si se observa en función del género, los chicos mantienen una participación equitativa a lo largo de las temporadas en las categorías con un descenso de participación en la cadete y, sobre todo, en la juvenil. Sin embargo, en la temporada 2013 hay una excepción en la categoría juvenil donde la participación se incrementa considerablemente $(7.5 \%)$ con la categoría infantil $(7.9 \%)$ y por encima del resto de categorías (alevín $7.4 \%$, benjamín $6.4 \%$ y cadete $5.9 \%$ ) como se muestra en la figura $5\left(\chi^{2}=44.48 ; \mathrm{p}<.001\right)$.

Figura 5. Participación de los chicos a lo largo de las tres temporadas en función de la categoría.

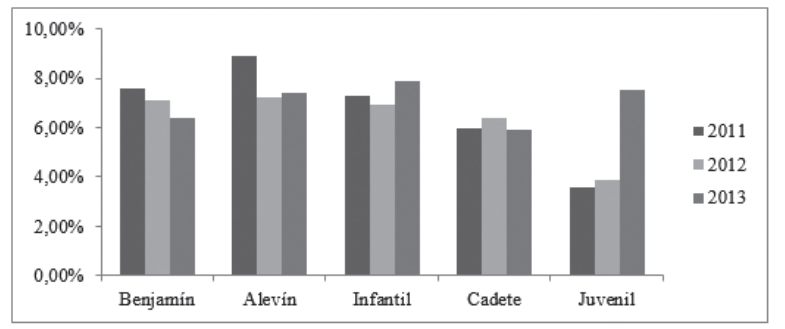

En el caso solo de las chicas, el criterio no es tan homogéneo $\left(\chi^{2}=185.28 ; \mathrm{p}<.001 ;\right.$ figura 6$)$. Sí existe la tendencia involutiva de participación, con diferencias estadísticamente muy significativas, más acentuada que en el género masculino. La categoría juvenil, seguida de la cadete, es la categoría donde menos participación hay (menos acentuada en el último año). En las categorías inferiores, en el año 2011 la más participativa en chicas fue la alevín (10.1\%), seguida de la benjamín (7.60\%) y después de la infantil (5.8\%). En 2012, cumpliendo con la tendencia involutiva, la mayor participación en chicas se produjo en la categoría benjamín (14.9\%), seguida de la alevín (8.2\%) y de la infantil (5.9\%). En la temporada 2013, la mayor participación fue en la categoría infantil (10.5\%), seguida de la alevín (7.6\%), y en esta temporada por primera 
vez, hay más participación en la cadete $(5.5 \%)$ y en la juvenil (4.7\%) que en la benjamín (4.2\%).

Figura 6. Participación de las chicas a lo largo de las tres temporadas en función de la categoría.

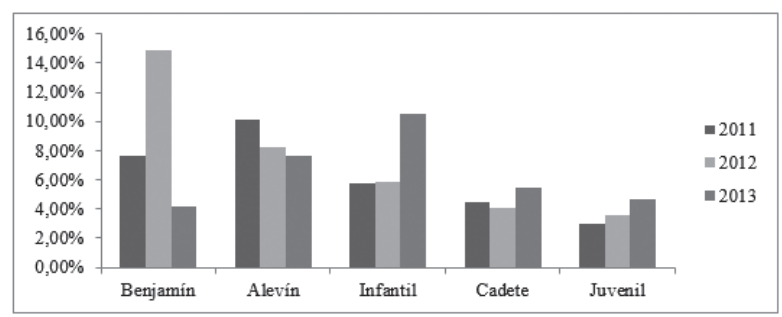

En función del tipo de centro, la mayoría de escolares participantes venían de centros públicos tanto en 2011 como en 2012 (33.6\% y 39.7\%, respectivamente), después de centros concertados con una participación del $13.7 \%$ en 2011 y $10.9 \%$ en 2012 y, por último, de centros privados $(1.3 \%$ en 2011 y $0.7 \%$ en 2012). Aunque estos valores se deban a la proporción de la muestra de centros educativos de Murcia, sí se puede decir que la participación de centros públicos aumenta mientras disminuye la de centros concertados y privados $\left(\chi^{2}\right.$ $=23.16 ; \mathrm{p}<.001$ ).

Si se compara la participación por género en función del tipo de centro, la participación tiende a ser muy equitativa, aunque en esta variable no se encuentra diferencias significativas considerando el tipo de centro y el género variables independientes.

\section{Discusión}

En atletismo se observa que conforme avanzan los escolares en edad escolar, abandonan la práctica deportiva, igual que sucede en la práctica de actividad física en general, siguiendo esta tendencia en la literatura revisada (Nuviala y Nuviala, 2005; Moreno, Martínez y Alonso, 2006; CSD, 2011; Isorna, Ruiz-Juan y Rial, 2013). Y aunque el total de los escolares que practican atletismo, como se comentó en el primer apartado, sea bajo (Chillón, Tercedor, Delgado y González-Gross, 2002; CSD, 2011), este deporte cumple requisitos y condiciones de las variables que influyen en adherencia a la práctica como observaron Cecchini, González, Carmona y Contreras (2004) cuando los escolares que lo practicaban cambiaban su orientación de meta hacia la tarea.

En 2010-11 y 2011-12 participaron más los benjamines y alevines, descendiendo la participación conforme avanzan en la categoría. Sin embargo, en 2013 la participación es más equitativa, aumentando la participación de cadetes y sobre todo juveniles con respecto a otras temporadas. Esto supone un aspecto muy positivo del cross escolar pues, en parte, puede ser por la labor de promoción de la federación, clubes, entrenadores, padres y/o centros educativos, que la promoción entre menores en esta última temporada es más equilibrada en todas las categorías, solventando un poco el problema de abandono que se lleva arrastrando años atrás como reflejan estos datos y gran parte de la literatura consultada (CSD, 2011). Estos datos parecen indicar que la nueva normativa sobre la competición de escolares federados en el cross y las tareas de promoción de las diferentes instituciones están consiguiendo sus propósitos.

Según el género, la participación es muy equitativa a lo largo de las temporadas. Esto se puede considerar un gran logro del cross escolar, pues en la mayoría de deportes escolares, en general, las chicas participan en menor proporción que los chicos (Moreno, Martínez y Alonso, 2006), y además, también abandonan la práctica deportiva en mayor proporción (CSD, 2011), mostrando los datos del presente estudio que en el cross escolar no hay diferencias en el abandono deportiva entre chicos y chicas en la Región de Murcia. También puede influir y podría ser interesante de analizar a los técnicos deportivos, Nuviala, León, Gálvez y Fernández (2007) observaron que la mayoría de técnicos deportivos eran hombres jóvenes.

Aunque estos datos estén sesgados por la mayor proporción tanto de la muestra como de los centros públicos ante los centros privados que hay en Murcia, sí se puede decir que la participación de centros públicos aumentó mientras disminuyó la de centros concertados y privados. Por tanto, las tareas de promoción deportiva tienen que tener en cuenta y orientar sus programas también a dichos centros.

Los chicos mantienen una participación equitativa por categorías con un descenso de participación en la cadete y, sobre todo, en la juvenil hasta la temporada 2012. Sin embargo, en la temporada 2013, aumenta la participación en las categorías superiores y la menor participativas se produce en las categorías benjamín y alevín. Algo similar sucede en las categorías superiores en las chicas, pues en las dos primeras temporadas tienen una mayor participación en las categorías inferiores que las superiores, sin embargo, en la temporada 2013 también hay un cambio significativo y es que hay una mayor participación en el resto de categorías. En este sentido, sería interesante saber si alguna campaña de promoción de aquellas temporadas fue más efectiva y por qué, viendo si esas participantes fueron las que mantuvieron su participación en la temporada siguiente una categoría más mayor.

\section{Conclusiones}

Con los resultados de este trabajo se pueden orientar los esfuerzos de la promoción deportiva en etapa escolar a los colectivos que más lo necesitan. En este sentido, se debe hacer un esfuerzo en la promoción del cross por parte de los esco- 
lares en las categorías superiores, evitando que abandonen la práctica deportiva. Según los datos de participación en chicas, se puede decir que se está haciendo un buen trabajo de promoción en el género femenino, sobre todo en las categorías inferiores. A partir de estos resultados de dónde focalizar los esfuerzos de promoción, hay que tener en cuenta cómo, poniéndose el énfasis en la diversión, el disfrute, el trabajo en equipo y el esfuerzo personal, por encima de la victoria y la competición, como señalaban Bailey, Cope y Pearce (2013).

El tipo de centro no influye en la participación de escolares pero sí sería interesante tener más en cuenta en la promoción deportiva a los centros concertados y privados, además de seguir promocionando el deporte entre los centros públicos.
Este estudio presenta algunas limitaciones. No se han tenido en cuenta, por ejemplo, el índice de natalidad de esas generaciones (se presupone una continuidad en la cantidad de escolares) ni se ha medido la efectividad o la influencia de la promoción del cross escolar (ya que el procedimiento de inscripción y las actividades de promoción de la FAMU seguían siendo iguales). Este estudio podría ayudar a focalizar los esfuerzos de promoción en el género y las categorías indicadas con menor participación y, además, crear campañas para intentar disminuir el abandono en la participación de las

últimas categorías indicadas.

\section{Referencias}

1. Bailey, R., Cope, E. J. y Pearce, G. (2013). Why do children take part in, and remain involved in sport? A literature review and discussion of implications for sports coaches. International Journal of Coaching Science, 1(7), 56-75.

2. Cecchini, J. A., González, C., Carmona, M. y Contreras, O. (2004). Relaciones entre el clima motivacional, la orientación de meta, la motivación intrínseca, la autoconfianza, la ansiedad y el estado de ánimo en jóvenes deportistas. Psicothema, 16, 104-109.

3. Chillón, P., Tercedor, P., Delgado, M. y González-Gross, M. (2002). Actividad físico-deportiva en escolares adolescentes. Retos, 1, 5-12.

4. CSD (2009). Plan Integral para la Actividad Física y el Deporte. Madrid: Consejo Superior de Deportes.

5. CSD (2011). Estudio de los hábitos deportivos de la población escolar en España. Madrid: Consejo Superior de Deportes.

6. González, S., García-López, L. M., Contreras, O. R. y Sánchez-Mora, D. (2009). El concepto de iniciación deportiva en la actualidad. Retos, 15, 14-20.

7. Gorgan, C. y Rata, G. (2013). Study regarding the selection and orientation of preparation of category in children, in middle distance running events at the national level. Sport si Societate. Revista de Educatie Fizica, Sport si Stiinte Conexe, 13(special issue), 129-132.

8. Isorna, M., Ruiz-Juan, F. y Rial, A. (2013). Variables predictoras del abandono de la práctica físico-deportiva en adolescentes. Ciencia, cultura y deporte, 23(8), 93-102.
9. Luengo, C. (2007). Actividad físico-deportiva extraescolar en alumnos de primaria. Revista Internacional de Medicina y Ciencias de la Actividad Física y el Deporte, 27(7), 174-184.

10. Mollá, M. (2007). La influencia de las actividades Extraescolares en los Hábitos deportivos de los Escolares. Revista Internacional de Medicina y Ciencias de la Actividad Física y el Deporte, 27(7), 241-252.

11. Moreno, J. A., Martínez, C., Alonso, N. (2006). Actitudes hacia la práctica físico-deportiva según el sexo del practicante. Revista Internacional de Ciencias del Deporte, 2(3), 20-43.

12. Norris, S. R. (2011). Learning to Train: A Key Stage in Developing Physical Literacy. Active Living, 3(22), 1-5.

13. Nuviala Nuviala, A. y Nuviala Nuviala, R. (2005). Abandono y continuidad de la práctica deportiva escolar organizada desde la perspectiva de los técnicos de una comarca aragonesa. Revista Internacional de Medicina y Ciencias de la Actividad Fisica y el Deporte, 19(5), 295-307.

14. Valovich, T. A., Decoster, L. C., Loud, K. J., Micheli, L. J., Parker, J T., Sandrey, M. A. et al. (2011). National Athletic Trainers' Association Position Statement: Prevention of Pediatric Overuse Injuries. Journal of Athletic Training, 46(2), 206-220.

15. Vílchez, M. P. (2013). Relación de la Educación Física con la práctica físico-deportiva de tiempo libre. Línea base de un estudio longitudinal comparativo en Costa Rica, México y España (tesis doctoral). Universidad Católica de Murcia (UCAM), Murcia. 